\title{
An Improved Phase Filter for Differential SAR Interferometry Based on an Iterative Method
}

\author{
Alejandro Mestre-Quereda, Student Member, IEEE, Juan M. Lopez-Sanchez ${ }^{(0)}$, Senior Member, IEEE, \\ Jesus Selva, and Pablo J. Gonzalez
}

\begin{abstract}
Phase quality is a key element in the analysis of the deformation of the Earth's surface carried out with differential synthetic aperture radar interferometry. Various decorrelation sources may degrade the surface deformation estimates, and thus, phase filters are needed for this kind of application. The wellknown Goldstein filter is the most widely used due to its simple implementation and computational efficiency. In the past years, improved filters have been proposed, which are based on this filter but introduce variations in the data processing. The effectiveness of these filters mostly depends on the size of the filtering window, the weight of the smoothed spectrum, and the kernel used to filter the spectrum. In this paper, we evaluate the performance of four of these filters and present a new method that outperforms all of them. The proposed filter is based on an iterative method in which the original phase is denoised progressively with adaptive filtering windows of different sizes. The effectiveness of the filter is controlled by the interferometric coherence, a direct indicator of the phase quality. Moreover, we introduce some modifications regarding the processing of the power spectrum. Specifically, we propose to smooth the original phase using a new filter which is based on a Chebyshev interpolation scheme. The performance of the new filter has been tested on both simulated and real interferograms, acquired by RADARSAT-2 and the Uninhabited Aerial Vehicle Synthetic Aperture Radar, which mapped two different geological events that caused surface deformation.
\end{abstract}

Index Terms-Differential synthetic aperture radar interferometry (InSAR), Goldstein filter, phase noise filtering.

\section{INTRODUCTION}

D IFFERENTIAL synthetic aperture radar interferometry (InSAR) is a powerful and established technique, which

Manuscript received December 1, 2017; revised February 7, 2018; accepted March 16, 2018. This work was supported in part by the Spanish Ministry of Economy, Industry and Competitiveness, in part by the State Agency of Research (AEI), in part by the European Funds for Regional Development under Project TIN2014-55413-C2-2-P and Project TEC2017-85244-C2-1-P, in part by the U.K. Natural Environmental Research Council through the Looking Inside the Continents under Grant NE/K011006/1, in part by the Rapid deployment of a seismic array in Ecuador following the April 16th 2016 M7.8 Pedernales earthquake under Grant NE/P008828/1, and in part by the Centre for the Observation and Modelling of Earthquakes, Volcanoes and Tectonics under Grant COMET, GA/13/M/031. (Corresponding author: Juan M. Lopez-Sanchez.)

A. Mestre-Quereda, J. M. Lopez-Sanchez, and J. Selva are with the Signals, Systems and Telecommunications Group, Institute for Computer Research (IUII), University of Alicante, E-03080 Alicante, Spain (e-mail: alejandro.mestre@ua.es; juanma.lopez@ua.es; jesus.selva@ua.es).

P. J. Gonzalez is with the Centre for the Observation and Modelling of Earthquakes, Volcanoes and Tectonics, Department of Earth, Ocean and Ecological Sciences, School of Environmental Sciences, University of Liverpool, Liverpool L69 3BX, U.K. (e-mail: pjgonzal@liverpool.ac.uk).

Color versions of one or more of the figures in this paper are available online at http://ieeexplore.iee.org.

Digital Object Identifier 10.1109/TGRS.2018.2820725 aims to detect and measure changes in the Earth's surface [1]-[5]. These changes, such as the ground deformation caused by a volcanic eruption or an earthquake, have been successfully monitored by exploiting the phase information contained in differential interferograms. The quality of the differential phase is very important for the subsequent analysis. Unfortunately, there exist different sources of decorrelation [6] (such as temporal decorrelation, volume decorrelation, and coregistration errors) that degrade the quality of the phase. In this context, interferometric phase filtering methods are mandatory in order to improve its quality. This improvement is especially needed for the phase unwrapping step [7], [8], which may be unfeasible with low-quality and noisy phase data. Filtering techniques also help recover phase measurements in areas in which otherwise it would be impossible. Nevertheless, a filtered interferogram has a lower effective spatial resolution and information is spatially correlated, but this caveat is acceptable considering the mentioned benefits on unwrapping and lower noise level.

Different interferogram filters have been proposed over the past years, being the well-known Goldstein filter [9], [10] the most common and widely used, as it offers good results in most cases and has a high computational efficiency. The Goldstein filter is a frequency-domain filter, which weights the 2-D Fourier transform of the complex interferogram signal by the absolute value of a smoothed version of itself. According to its original formulation, the filter divides the original interferogram into overlapped windows of a fixed size. The spectrum is smoothed in the frequency domain by the convolution with a Boxcar or Gaussian kernel. Moreover, the effectiveness of the filter is controlled by a real parameter defined between zero and one, commonly named $\alpha$, that is kept constant for the whole interferogram. This parameter governs the filtering strength and has to provide a tradeoff between noise reduction and resolution loss.

An $\alpha$-adaptive filter was proposed in [11]. In this modified Goldstein filter, the values of $\alpha$ are selected as a function of the interferometric coherence, a commonly used indicator of the phase quality. Low-coherent (decorrelated) areas will then be filtered with higher values of $\alpha$, whereas high-coherent interferogram parts will be filtered with lower values of $\alpha$, i.e., less filtered. The adaptivity of the $\alpha$ parameter to the nonuniform distribution of the noise has improved the fringe continuity preservation and limited the loss of resolution caused by high values of $\alpha$ [11].

Another important parameter of the Goldstein filter and its modified version is the selected window size. Larger 
filtering windows provide a better result in terms of noise reduction but at the expenses of a resolution loss based on [11]. Suo et al. [12] introduced a new method in which each pixel is filtered individually. The window size employed to filter each pixel in the interferogram is adaptively computed as a function of the pixel's coherence and the phase standard deviation of the whole interferogram. The results obtained show a very good performance in terms of fringe continuity preservation even in very dense fringe areas, but noise reduction is not enough in areas with very noisy data.

Alternatively, a different strategy was proposed in [13] and [14]. The method consists in iteratively filtering the original interferogram with windows of decreasing size. In each iteration, the coherence-adaptive Goldstein filter is applied and the window size is reduced by half. That filter displays a dramatic noise reduction and allows us to recover useful measurements even with very noisy original interferometric data. However, the main drawback of that filter is that the detailed features of the deformation pattern might be lost due to the repeated use of large filtering windows, especially in the first iterations of the algorithm.

In this paper, a new interferometric phase filtering method is proposed. The filter relies on the iterative and coherenceadaptive Goldstein approach [14], but an improved methodology has been applied. As in that filter, the filtering parameter $\alpha$ is selected locally as a function of the coherence. However, the spectrum smoothing is substituted by a filtering operation which is performed in the spatial domain by means of adaptive kernels based on Chebyshev polynomials of a given order. According to the different filtering problems previously mentioned, the main objectives of the proposed method are: 1) to effectively suppress the phase noise in wide areas, i.e., lowfrequency areas where weak phase gradients are present and 2) to preserve strong gradient information in small areas related to surface deformation patterns caused by a geological event.

This paper is organized as follows. Section II presents a revision of the formulation of the original Goldstein filter and the existing improved versions. In Section III, we detail the different modifications that have been made to improve the filtering quality. In Section IV, we evaluate the results both for simulated data with different noise levels and real SAR data mapping two geological events. Specifically, the proposed algorithm has been tested with RADARSAT-2 data mapping the Mount Etna's volcanic eruption (Sicily, Italy) in May 2008 and data acquired from the airborne Uninhabited Aerial Vehicle Synthetic Aperture Radar (UAVSAR) corresponding to the Kilauea's volcanic eruption (HI, USA) in March 2011. Finally, the main conclusions are summarized in Section V.

\section{Discussion ON THE GOLDSTEIN FILTER AND ITS VARIANTS}

\section{A. Review of the Original Goldstein Filter}

Initially proposed in [9], the Goldstein filter divides the original noisy interferogram into small windows, or blocks, of a fixed size. The spectrum $\boldsymbol{S}_{f}$ of the filtered interferogram is obtained as

$$
\left[\boldsymbol{S}_{f}\right]_{f_{x}, f_{y}}=\left|\left[\boldsymbol{S}_{m}\right]_{f_{x}, f_{y}}\right|^{\alpha} \cdot[\boldsymbol{S}]_{f_{x}, f_{y}}
$$

where $S$ is the original spectrum of the interferogram window, $\left|\boldsymbol{S}_{m}\right|$ is the absolute value of the smoothed spectrum, $\left(f_{x}, f_{y}\right)$ are the 2-D spatial frequencies (in the azimuth and range directions), and symbol · denotes the Hadamard or elementby-element product. Parameter $\alpha$ is a real number defined in the $[0,1]$ interval and has a big impact on the effectiveness of the final filtering, since it defines the weight of the smoothed spectrum with respect to the original spectrum. For instance, if $\alpha=0$, the filtered spectrum will be equal to the original spectrum (i.e., no filtering occurs), but the filtering becomes more significant as the values of $\alpha$ increase. Moreover, the window size is generally selected as a power of $2(32 \times 32,64 \times 64$ pixels $\ldots)$, which accelerates the $2-\mathrm{D}$ fast Fourier transform computation of the spectrum. In order to avoid discontinuities at the boundaries between the blocks, two adjacent filtering windows are usually overlapped.

The smoothing operation is achieved by a convolution of the original power spectrum with a predefined square kernel, such as a mean or a Gaussian kernel of a certain size (e.g., $5 \times 5$ or $7 \times 7$ ).

The main drawback of this algorithm is related to the selection of the filter parameter $\alpha$, as it is chosen manually (e.g., based on a visual inspection of the original noisy phase), and it is kept constant for the whole interferogram. As stated before, noisy interferograms would benefit from larger values of $\alpha$, but fixing a value of this parameter close to 1 could cause a resolution loss, especially in dense fringe areas. Consequently, a value of $\alpha=0.5$ is usually employed to ensure a balance between noise reduction and fringe preservation.

\section{B. Adaptive Goldstein Filters}

In practice, some areas in the interferogram are more affected by noise than others, i.e., noise is not uniformly distributed in the scene. The use of a single filtering parameter $(\alpha)$ leads to a nonoptimum noise reduction, as noisy areas should be strongly filtered and high-quality areas should not be overfiltered. Baran et al. [11] proposed an $\alpha$-adaptive Goldstein filter in which the value of $\alpha$ in each filtering window is computed as a function of the interferometric coherence, a commonly used indicator of the phase quality. Coherence $\gamma$ quantifies the correlation between the two combined SAR acquisitions and can be estimated as [15]

$$
\gamma=\frac{E\left\{\boldsymbol{S}_{1} \boldsymbol{S}_{2}^{*}\right\}}{\sqrt{E\left\{\boldsymbol{S}_{1} \boldsymbol{S}_{1}^{*}\right\} \cdot E\left\{\boldsymbol{S}_{2} \boldsymbol{S}_{2}^{*}\right\}}}
$$

where $\boldsymbol{S}_{1}$ and $\boldsymbol{S}_{2}$ are the two images forming the interferogram and $E\{\}$ corresponds to the mathematical expectation which, in practice, is replaced by a spatial average inside an estimation window. The module $|\gamma| \in[0,1]$ of the coherence is usually employed to characterize the phase noise of the interferogram.

The probability density function of the phase $\phi$ was derived in [16] as a function of the coherence $\gamma$ and the number of 


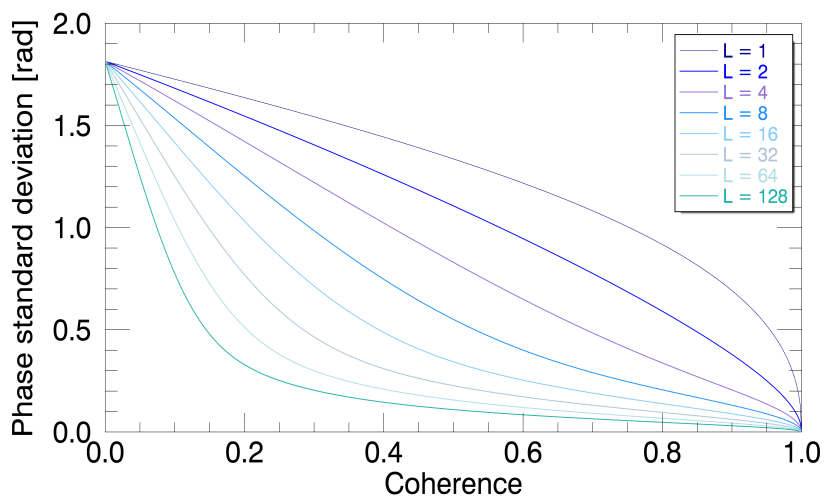

Fig. 1. Phase standard deviation as a function of coherence and a number of looks.

looks $L$ used in its estimation

$$
\begin{aligned}
\operatorname{PDF}(\phi)= & \frac{\Gamma(L+1 / 2)\left(1-|\gamma|^{2}\right)^{L} \gamma \cos \left(\phi-\phi_{0}\right)}{2 \sqrt{\pi} \Gamma(L)\left(1-|\gamma|^{2} \cos ^{2}\left(\phi-\phi_{0}\right)\right)^{L+1 / 2}} \\
& \quad+\frac{\left(1-|\gamma|^{2}\right)}{2 \pi} F_{1}\left(L, 1 ; 1 / 2 ; \gamma^{2} \cos ^{2}\left(\phi-\phi_{0}\right)\right)
\end{aligned}
$$

where $\Gamma$ is the gamma function, $F_{1}$ is the Gauss hypergeometric function, and the expected phase $\phi_{0}$ can be set to zero without loss of generality. The phase standard deviation $\sigma_{\phi}$ can be computed as

$$
\sigma_{\phi}=\sqrt{\int_{\phi}\left(\phi-\phi_{0}\right)^{2} \operatorname{PDF}(\phi) d \phi} .
$$

The original Goldstein formulation was modified in [11] by setting

$$
\alpha=1-|\bar{\gamma}|
$$

where $|\bar{\gamma}|$ is the mean coherence of the effective filtering window. Note that the effective window (nonoverlapped area) is employed to compute $|\bar{\gamma}|$ in order to prevent the mean coherence from being affected by coherence values from the overlapped areas. Consequently, low-coherence parts of the interferogram are strongly filtered, as the values of $\alpha$ are larger, and high-coherence parts are filtered less.

In addition to the adaptive values of the filtering parameter $\alpha$, a window-adaptive filter has been recently proposed in [12]. Each pixel in the interferogram is filtered individually, and the window size used for each pixel is computed as a function of the local coherence and the phase standard deviation of the whole scene.

Based on (3) and (4), Fig. 1 shows the phase standard deviation as a function of the coherence for a different number of looks. As explained in [12], the window size selected to filter a pixel corresponds to the square root of the multilook number provided by the crossing in Fig. 1 of the coherence and the standard deviation. For instance, if a pixel's coherence is 0.4 and the computed phase standard deviation of the interferogram is 0.5 , the crossing point corresponds to a number of looks equal to 16 . Then, the pixel will be filtered with a $\sqrt{16}$ size, i.e., a $4 \times 4$ window [12].

In addition, the filter proposed in [12] introduces some modifications regarding the power spectrum processing. First, the local phase gradients in the filtering window are extracted prior to the filtering step. The local estimation of the phase ramp allows us to center the spectrum and to obtain the dominant frequencies inside the filtering window, which is useful for resolution preservation. This step will be explained in more detail in Section III-B.

Then, the spectrum of the slope-compensated samples in the filtering window is multiplied by a 2-D sinc function [12]. Finally, the filtering parameter is modified to

$$
\alpha=1-|\gamma|^{2}
$$

where $|\gamma|$ is the pixel coherence. This modification increases the degree of filtering with respect to Baran's modified filter if the same window size is used. However, oversmoothing is avoided, since the size of the filtering window is small for high-coherence pixels, as shown in Fig. 1.

In general, both adaptive filters show good results, especially concerning the preservation of fringe details. It has been proven that the pixel-by-pixel strategy preserves the resolution of the original phase very clearly even in highdensity fringe areas. Nevertheless, in the case of very noisy interferometric phases, none of these filters show good results in noise reduction. The coherence-adaptive Goldstein filter would need larger windows in order to effectively suppress the noise at the cost of a resolution loss proportional to the size of the filtering window. Large windows provide a better estimate of the power spectrum of the interferogram patch, as a large number of pixels are considered in the estimation. Therefore, the smoothing operation is more significant, i.e., the filter is better adapted to the signal. Furthermore, the size of each filtering window in the pixel-by-pixel filter might not be big enough. An important issue related to this filtering strategy is that the adaptive window sizes are computed with regard to the phase standard deviation of the whole scene. Unfortunately, a single value of phase standard deviation does not reflect correctly the noise level in all areas of the interferogram, as noise is not uniformly distributed.

\section{Recursive Adaptive Spectral Filter}

In order to effectively suppress the phase noise with the Goldstein filter, the combination of large filtering windows and high values of the filtering parameter $\alpha$ is needed. An interesting strategy for this purpose is to filter repeatedly the interferogram. This methodology has been proposed in [14] and is named recursive adaptive spectral filter (RASF). The core idea consists in applying the coherence-adaptive Goldstein filter with filtering windows of decreasing size in an iterative fashion. Since noise suppression is more effective with larger filtering windows, large windows are employed in the first iterations of the algorithm (for instance, the initial size could be $256 \times 256$ pixels). In the subsequent iterations, the window size is divided in half until it reaches the minimum size, for instance, an $8 \times 8$ window. Additionally, a modification concerning the filtering parameter is proposed. In this case, the degree of correlation between the two combined SAR images is estimated with phase values only. The phase-only coherence estimate, or phase coherence $\rho$, can be computed 


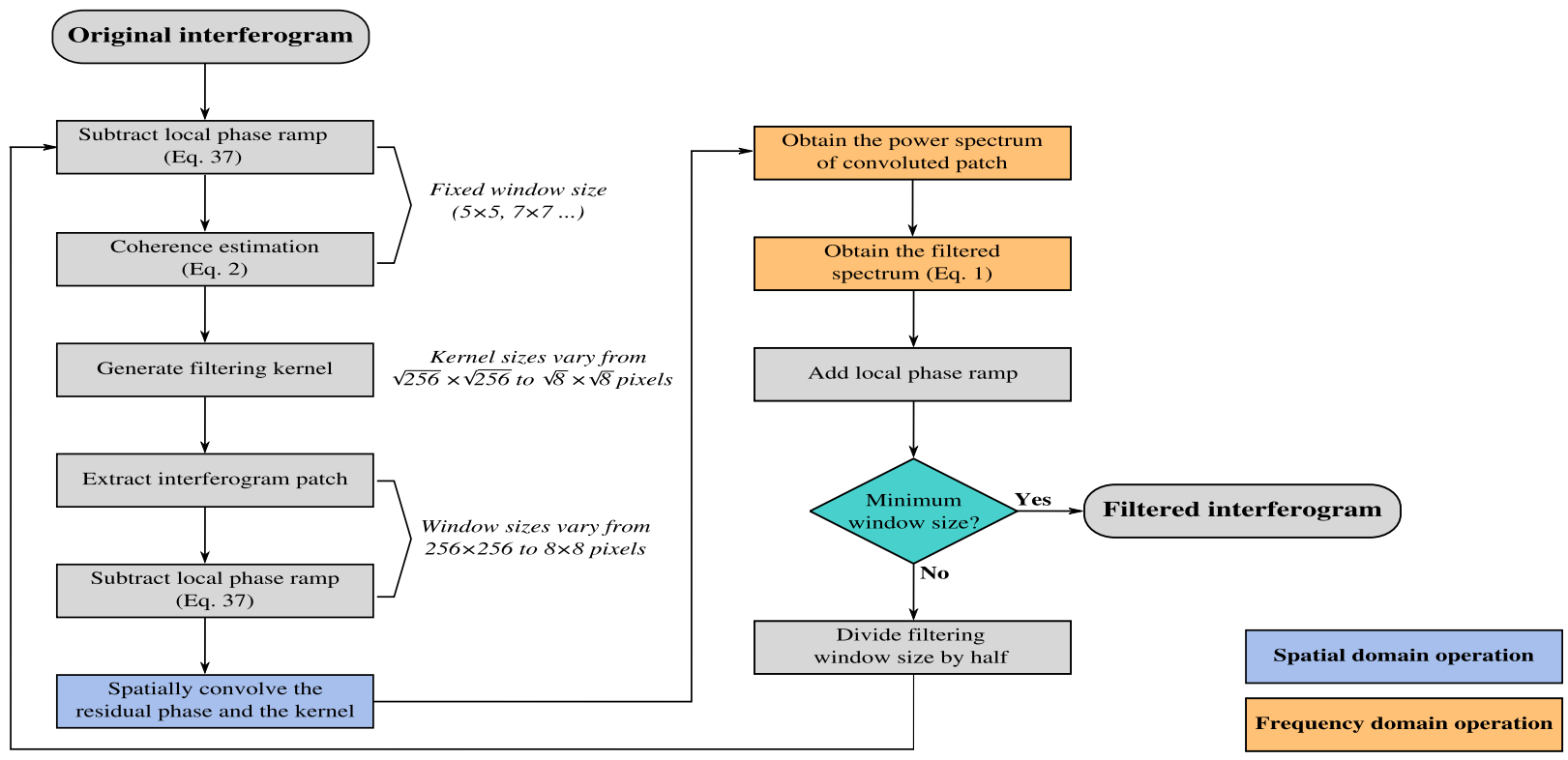

Fig. 2. General scheme of the proposed filter.

by means of a spatial average inside an estimation window with $M$ samples as

$$
\rho=\frac{1}{M} \sqrt{\sum_{m=0}^{M-1} \cos ^{2}\left(\phi_{m}\right)+\sum_{n=0}^{M-1} \sin ^{2}\left(\phi_{m}\right)}
$$

where $\phi_{m}$ is the $m$ th phase sample in the estimation window. As explained in [14], two different phase coherence maps are computed at each iteration. The final correlation $\hat{\rho}$ value from which the filtering parameter $\alpha=1-\hat{\rho}$ will be calculated is

$$
\hat{\rho}= \begin{cases}\rho-\Delta \rho, & \text { if } \rho^{*}>\rho \\ \rho^{*}, & \text { otherwise }\end{cases}
$$

where $\rho$ is the original phase coherence, $\rho^{*}$ is the phase coherence after subtraction of the local phase ramp, and $\Delta \rho=\rho^{*}-\rho$.

The subtraction of the local phase ramp will increase the values of the original phase coherence, i.e., $\rho^{*}>\rho$. Then, $\rho-\Delta \rho<\rho$ and, consequently, the values of $\alpha$ will be larger in comparison with the ones of the modified Goldstein filter. Therefore, the resulting phase is likely to be more filtered. This iterative filtering strategy has proved to be very effective in noise reduction even with extremely noisy interferograms [14].

\section{Proposed Method}

The general scheme of the filter proposed in this paper is represented with the block diagram of Fig. 2. The main steps will be explained in detail in Sections III-A-III-C.

\section{A. Kernel Generation}

Conventionally, the Goldstein-filtered interferogram is obtained by the 2-D inverse fast Fourier transform (2-D IFFT) of the product of the smoothed spectrum and the original spectrum, that is, the 2-D IFFT of (1). The smoothing operation is also performed in the frequency domain by means of a predefined kernel, such as a Gaussian kernel or a mean kernel of a fixed size. In our method, the smoothing operation is applied in the spatial domain. Then, the original spectrum is weighted by the spectrum of the smoothed block by means of (1) as in the Goldstein filter case.

Furthermore, we propose a specific filtering kernel that is derived from the combination of a Chebyshev interpolator and the pseudoinverse. In order to derive this kernel, we start by considering the approximation of a generic 1-D complex function $F(x)$ and then draw several results that finally lead to a 2-D interpolation kernel for a generic 2-D function. Since the derivation is somewhat long, we first state its main steps and then justify them in detail.

Thus, we start by considering a function $F(x)$, defined in $[-1,1]$, and assume that its value is approximately known at $N$ abscissas $x_{p}$, i.e., we assume that a set of values $\hat{f}_{p}$ is available such that

$$
\hat{f}_{p} \approx F\left(x_{p}\right), \quad(p=1,2, \ldots, N)
$$

for $N$ distinct abscissas $x_{p}$ in $[-1,1]$.

Next, we introduce an interpolator and perform other operations in several steps in order to obtain the convolution kernel. Step 4 is where we obtain such a kernel. The steps are as follows.

1) We assume that $F(x)$ can be well approximated by a Chebyshev sum of order $K_{x}$ of the form

$$
F(x) \approx \sum_{k=0}^{K_{x}-1} c_{k} T_{k}(x)
$$

where the prime (') indicates that the $k=0$ summand must be multiplied by $1 / 2, c_{k}$ is a set of unknown coefficients, and $T_{k}(x)$ is the Chebyshev polynomial of the first kind and order $k$

$$
T_{k}(x) \equiv \cos (k \arccos x) .
$$


2) Using (9) and the model in (10), we estimate the set of coefficients $c_{p}$ using the pseudoinverse of the linear system implicit in (10).

3) For any $x$, we estimate $F(x)$ by inserting in (10) the coefficients $c_{p}$ obtained in the previous step.

4) If we fix a specific $x$ and the abscissas $x_{p}$ are regularly spaced, then the previous step delivers a convolution kernel, given that (10) depends linearly on the coefficients $c_{p}$.

5) All the previous steps can also be applied to a function $G(z)$ varying in a generic interval $[a, b]$, simply by introducing the change of variable

$$
x=-1+2 \frac{z-a}{b-a} .
$$

6) All the previous steps can be extrapolated to a 2-D function in a straightforward way and, therefore, they allow us to derive a 2-D kernel for a generic 2-D function $G\left(z_{1}, z_{2}\right)$.

Let us justify these steps. In step 1 , we have interpolated $F(x)$ using a Chebyshev sum with $K_{x}$ coefficients. This interpolator is well known in the approximation theory for its excellent performance. Actually, its minimax error is close to that of the minimax polynomial [17, Th. 6.13], and the interpolator converges to $F(x)$ as $K_{x}$ increases just assuming that the function is continuous [17, Th. 6.5]. Besides, it allows us to view $F(x)$ as a signal whose spectrum is the set of coefficients $c_{k}$. To see this point, we must recall the basic variable change in Chebyshev interpolation, which is

$$
x=\cos \theta, \quad \theta \in[0, \pi] .
$$

In terms of $\theta,(10)$ is

$$
F(\cos \theta) \approx \sum_{k=0}^{K_{x}-1} c_{k} \cos \left(k \theta_{p}\right)
$$

where

$$
\theta_{p} \equiv \arccos x_{p}
$$

Thus, we are viewing the function as a cosine polynomial. Note that since the cosine is an even function, if $F(x)$ is continuous, then $F(\cos \theta)$ is continuous at all $\theta$. This fact mitigates the Gibbs phenomenon that usually occurs at the interval edges when a function is being approximated with a set of polynomials. Note that this is related to the approximation theory, and it is not related to the inclusion of an overlap between the interferogram patches in the implementation of the filter.

In step 2, we use the pseudoinverse to estimate the coefficients $c_{k}$. Specifically, we first combine (9) and (10) into the linear model

$$
\hat{f}_{p} \approx \sum_{k=0}^{K_{x}-1} c_{k} T_{k}\left(x_{p}\right)
$$

which can be written in the matrix form as

$$
\hat{f} \approx T_{c}
$$

where

$$
[\hat{\boldsymbol{f}}]_{p} \equiv f_{p},[\boldsymbol{T}]_{p, .} \equiv \boldsymbol{t}\left(x_{p}\right),[\boldsymbol{c}]_{k} \equiv c_{k}
$$

and

$$
\boldsymbol{t}(x) \equiv \begin{cases}T_{k}(x), & k>0 \\ 1 / 2, & k=0 .\end{cases}
$$

Finally, the pseudoinverse provides the estimate of $\boldsymbol{c}$

$$
\hat{c} \equiv \boldsymbol{T}^{\dagger} \hat{\boldsymbol{f}}
$$

that minimizes in the coefficients $c_{k}$ the quadratic error

$$
\sum_{p=1}^{N}\left\|\hat{f}_{p}-\sum_{k=0}^{K_{x}-1} c_{k} \cos \left(k \theta_{p}\right)\right\|^{2} .
$$

If there is only one vector $\hat{\boldsymbol{c}}$ minimizing this error, then the pseudoinverse delivers it but if there is more than one $\hat{\boldsymbol{c}}$ minimizing (21), then it delivers the one among them with minimum energy, i.e., the one for which $\|\hat{\boldsymbol{c}}\|^{2}$ is minimum. This last feature is useful whenever there all less data samples $\hat{f}_{p}$ than coefficients $c_{k}$ given that, in this case, the pseudoinverse delivers the "simplest" set $\hat{c}$ in the sense that its energy is minimum.

In step 3, we estimate $F(x)$ at any $x$ using $\hat{\boldsymbol{c}}$. This estimator is obtained by replacing $c_{k}$ with the elements of $\hat{\boldsymbol{c}}$ in (10). Its matrix form is

$$
\hat{F}(x) \equiv \boldsymbol{t}(x)^{T} \hat{\boldsymbol{c}}=\boldsymbol{t}(x)^{T} \boldsymbol{T}^{\dagger} \hat{\boldsymbol{f}} .
$$

Step 4 is a particular case of step 3, in which we take $x=0$ and assume that the abcissas $x_{p}$ are regularly spaced and sorted in the increasing order. In this case, we have that the vector multiplying $\hat{f}$ in (22) is a reversed convolution kernel. In other words, if the sequence $x_{p}$ is infinite, then we may filter it using the kernel

$$
\left[\boldsymbol{k}_{c h}\right]_{p} \equiv\left[\boldsymbol{t}(0)^{T} \boldsymbol{T}^{\dagger}\right]_{N-p+1} .
$$

Step 5 is straightforward, given that we may estimate the function

$$
F(x)=G\left(\frac{b+a}{2}+\frac{b-a}{2} x\right) .
$$

We may now repeat the previous five steps for the 2-D case and obtain similar results (step 6). Given a two-variable function $F(x, y)$, defined in $[-1,1]$ for both variables, and a set of samples $\hat{f}_{p}$ taken at abscissas $x_{p}, y_{p}, p=1,2, \ldots, N$, we start by introducing a bivariate Chebyshev model for $F(x, y)$

$$
F(x, y) \approx \sum_{k=0}^{K_{x}-1} \sum_{r=0}^{K_{y}-1} c_{k, r} T_{k}(x) T_{r}(y)
$$

and posing the linear system

$$
\hat{f}_{p} \approx \sum_{k=0}^{K_{x}-1} \sum_{r=0}^{K_{y}-1} c_{k, r} T_{k}\left(x_{p}\right) T_{r}\left(y_{p}\right) .
$$

In the matrix form, this system reads

$$
\hat{\boldsymbol{f}} \approx \boldsymbol{T}_{2 D} \boldsymbol{c}_{2 D}
$$


where

$$
[\hat{\boldsymbol{f}}]_{p} \equiv f_{p}, \quad\left[\boldsymbol{c}_{2 D}\right]_{k+(r-1) K_{x}} \equiv c_{k, r}
$$

and

$$
\left[\boldsymbol{T}_{2 D}\right]_{p, k+(r-1) N_{x}} \equiv\left[\boldsymbol{t}_{x}\left(x_{p}\right)\right]_{k}\left[\boldsymbol{t}_{y}\left(y_{p}\right)\right]_{r} .
$$

The pseudoinverse gives the coefficients' estimate

$$
\hat{\boldsymbol{c}}_{2 D} \equiv \boldsymbol{T}_{2 D}^{\dagger} \hat{\boldsymbol{f}}
$$

and the estimate $\hat{F}(0,0)$ is given by

$$
\hat{F}(0,0) \equiv \boldsymbol{t}_{2 D}(0,0)^{T} \boldsymbol{T}_{2 D}^{\dagger} \hat{\boldsymbol{f}}
$$

where

$$
\left[\boldsymbol{t}_{2 D}(x, y)\right]_{k+K_{x}(r-1)} \equiv\left[\boldsymbol{t}_{x}(x)\right]_{k}\left[\boldsymbol{t}_{y}(y)\right]_{r}
$$

and where $\boldsymbol{t}_{x}(x)$ and $\boldsymbol{t}_{y}(y)$ have respective lengths $K_{x}$ and $K_{y}$, and follow the definition in (19).

Finally, assume that the abscissas $\left(x_{p}, y_{p}\right)$ are those in a regular grid of size $N_{x} \times N_{y}$ and $N=N_{x} N_{y}$ (this size corresponds to the size of the convolution kernel). These abscissas follow the equations:

$$
\begin{aligned}
& x_{p}=x_{0}+\Delta x(\alpha(p)-1) \\
& y_{p}=y_{0}+\Delta y(\beta(p)-1)
\end{aligned}
$$

for a fixed position $\left(x_{0}, y_{0}\right)$ and positive increments $\Delta x$ and $\Delta y$, where $\alpha(p)$ and $\beta(p)$ are the only nonnegative integers, such that $p=\alpha(p)+N_{x}(\beta(p)-1)$ with $0 \leq \alpha(p)<N_{x}$.

For this grid, we obtain from (31) the convolution kernel

$$
[\boldsymbol{K}]_{\alpha+1, \beta+1} \equiv\left[\boldsymbol{t}_{2 D}(0,0)^{T} \boldsymbol{T}_{2 D}^{\dagger}\right]_{N-\left(\alpha+N_{x} \beta\right)}
$$

where $\alpha=0,1, \ldots, N_{x}-1$ and $\beta=0,1, \ldots, N_{y}-1$.

In practice, we assume that the convolution kernel is square, i.e., we impose $N_{x}=N_{y}$ and the polynomial order in both the dimensions is the same $\left(K_{x}=K_{y}\right)$. Both the size of the kernel and the polynomial order have an impact on the filtering results; however, the size of the convolution kernel is more significant. Larger kernels will provide stronger filtering. For this reason, the size of the convolution kernel will vary in each filtering iteration as a function of the patch size. In Section III-C, a more detailed explanation about each window size will be given. Concerning the polynomial order, lower values provide slightly more low-pass filtering. However, we have verified that this has a minor impact on the final filtering results.

According to the previous formulation, an example of an $11 \times 11$ convolution kernel is shown in Fig. 3. The polynomial order in both the dimensions has been fixed to 20 .

\section{B. Local Fringe Frequencies' Estimation}

The terrain deformation caused by a geological event can be visually observed in the differential interferogram by dense fringe areas (for instance, around the volcano crater after an eruption). It is known that the presence of interferometric fringes affects the estimation of the correlation (coherence) between the SAR acquisitions [15]. Consequently, any bias

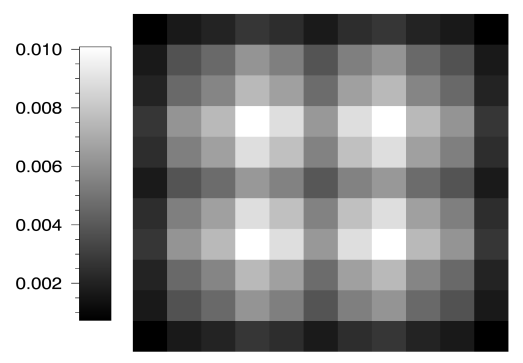

Fig. 3. Example of an $11 \times 11$ convolution kernel based on 2-D Chebyshev polynomials of 20 orders.

should be corrected before its estimation as in the context of InSAR.

As detailed in [18], the phase of a complex differential interferogram can be locally modeled by a 2-D sine function. Then, inside a $(2 \mathrm{P}+1) \times(2 \mathrm{Q}+1)$ window, the phase signal presents the following form:

$$
Z_{p, q}=e^{2 \pi j\left(f_{x} p+f_{y} q\right)}
$$

where $\left\{f_{x}, f_{y}\right\}$ are the local 2-D frequencies at indexes $(p, q)$ and noise is omitted for simplicity. The dominant 2-D frequencies inside the window centered on pixel $\left(p_{0}, q_{0}\right)$ are estimated with the maximum likelihood method [19] in the spectral domain

$$
\left\{\hat{f}_{x}, \hat{f}_{y}\right\}=\max _{f_{x}, f_{y}}\left(\left|\sum_{x=p_{0}-P}^{p_{0}+P} \sum_{y=q_{0}-L}^{q_{0}+Q} \boldsymbol{Z}_{p, q} e^{-2 \pi j\left(f_{x} p+f_{y} q\right)}\right|\right) .
$$

The main phase component $\hat{\theta}_{0}$ can be estimated as the Fourier transform of $z$ evaluated at $\left\{\hat{f}_{x}, \hat{f}_{y}\right\}$ spatial frequencies. Finally, the slope-compensated phase values $\hat{z}$ at pixels $(p, q)$ are expressed as

$$
\hat{\boldsymbol{Z}}_{p, q}=z_{p, q} e^{-2 \pi j\left[\left(\hat{f}_{x} p+\hat{f}_{y} q\right)-\hat{\theta}_{0}\right]} .
$$

Notice that, without the presence of noise, the values of $\hat{z}$ tend to zero, as any residual phase gradient has been removed and any phase offset has also been subtracted from the original phase values.

\section{Modified-Coherence-Adaptive and Iterative Filtering}

In order to meet the objectives already mentioned, i.e., noise suppression in wide and low-frequency areas and resolution preservation in dense fringe zones, different modifications regarding the filtering parameters are proposed.

As in the RASF, an iterative and adaptive filtering strategy is proposed. We filter the original interferogram a number of times with decreasing filtering windows. Large windows are employed in the first filter iterations, for instance, a $256 \times 256$ window. The initial size is an input parameter of the algorithm. At each iteration, the filtering window size is reduced by half until a minimum size of $8 \times 8$ pixels is reached. The filtering kernel is also adaptive, so it varies in each iteration of the algorithm. The size of the kernel is the 
square root of the size of the filtering window. Then, it varies from $\sqrt{256} \times \sqrt{256}$ to $\sqrt{8} \times \sqrt{8}$ (rounded to nearest integer).

Additionally, the subtraction of the local phase ramp by means of (37) and (38) is employed in two different steps of the proposed method. First, local fringes are removed from the original phase prior to coherence estimation. In this regard, the removal of the local phase ramp causes an increase in the correlation, and then, coherence values provide a more accurate estimation of the noise level. This coherence will be used to compute the values of the filtering parameter $\alpha$ in each iteration. In this step, conventional window sizes for coherence estimation are used, for instance, $5 \times 5$ or $7 \times 7$ windows. Note that, as coherence increases, the values of $\alpha$ will be smaller than in other methods, but the iterative filtering will progressively denoise the original phase. Moreover, the local fringes are also removed from the original signal prior to the filtering step, i.e., when an interferogram patch is extracted. Then, only the spectrum of the remaining values (which are considered as noise) is filtered, so the information related to the deformation fringes is better preserved.

\section{Results}

Different data sets have been used to validate the effectiveness of the proposed method. We first evaluate the algorithm with four simulated interferograms in which different levels of noise have been added. The performance of the proposed method is compared quantitatively with the different filters described in Section III. The capability of the proposed filter is also tested with real SAR data corresponding to two different geological events and SAR sensors.

\section{A. Simulated Scenes With Different Noise Levels}

The simulated phases are obtained as the sum of a smooth 2-D function corresponding to the peaks function in MAT$\mathrm{LAB}$ and a function with a high gradient which corresponds to a 2-D arctangent function. This high gradient function will be especially useful to detect overfiltering effects. The simulated phase is shown in Fig. 4. The size of the scene is $1000 \times 1000$ pixels.

According to (3) and (4), four different noise levels are added to the original phase (see Table I for details). As noise is randomly generated, we ran each simulation case over 100 times. For the sake of simplicity, noise is uniformly added in the original data by fixing the number of looks $L=9$ and varying the mean coherence $|\bar{\gamma}|$. The standard deviation of the noise varies between 0.509 and $2.569 \mathrm{rad}$. Note that the largest phase standard deviation has been manually added to test the performance of every method in an extremely noisy scene.

The performance of each filter is assessed by the number of residues [7] and the mean square error (MSE), which is defined as [19]

$$
\operatorname{MSE}=E\left\{\left|\arg \left(e^{j\left(\phi_{F}-\phi_{\text {ideal }}\right)}\right)\right|^{2}\right\}
$$

where $\phi_{F}$ is the filtered phase and $\phi_{\text {ideal }}$ is the noise-free phase. As each simulated scene is generated multiple times, the number of remaining residues and MSE values that we

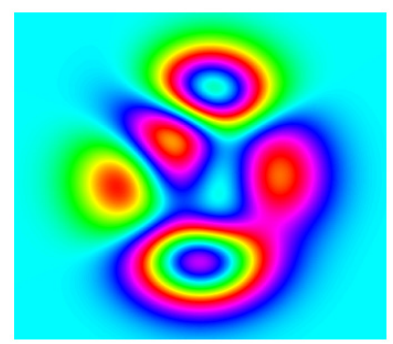

(a)

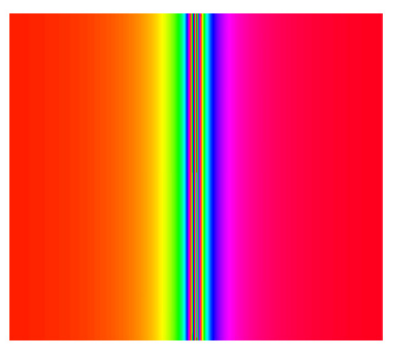

(b)

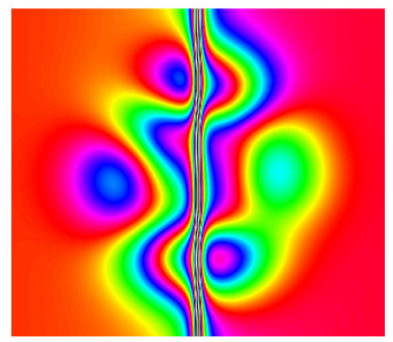

(c)

Fig. 4. Noise-free simulated phase. (a) Smooth 2-D Gaussian function. (b) Variable and increasing phase. (c) Final simulated phase.

TABLE I

CHARACTERISTICS OF THE Four Simulated INTERFEROgRAMS

\begin{tabular}{cccc}
\hline Interferogram & $|\bar{\gamma}|$ & $L$ & $\sigma_{\phi}[\mathrm{rad}]$ \\
\hline$I_{1}$ & 0.50 & 9 & 0.509 \\
$I_{2}$ & 0.30 & 9 & 0.941 \\
$I_{3}$ & 0.15 & 9 & 1.367 \\
$I_{4}$ & 0.075 & 9 & 2.569 \\
\hline
\end{tabular}

present in this section corresponds to the mean values of all the realizations.

It is important to point out that in order to correctly compare the performance of all filters, the same parameters have been employed when possible. Concerning the Goldstein filter, its adaptive version, and the RASF, the smoothing operation consists in a convolution with a $7 \times 7$ Gaussian kernel. The spatial bandwidth of the Gaussian kernel is 2.5. Regarding the pixel-by-pixel algorithm, the power spectrum is weighted with a $3 \times 3$ sinc function. In our proposed method, the Chebyshev-polynomial-based kernel is adaptive with regard to the filtering window size, and the polynomial order is fixed to 20. In addition, the overlap between two adjacent filtering windows is set to $3 / 4$ of the size of the filtering window for all block-filtering methods. The rest of the filtering parameters are summarized in Table II. Note that a patch size of $256 \times 256$ has been used in both the original Goldstein and adaptive Goldstein filters. This patch size is larger than the one proposed in the original publications [9], [11], which is $32 \times 32$ pixels. The reason of using this patch size is to enhance the result provided by both conventional methods. As stated previously, larger filtering windows offer better results in noise reduction. Consequently, filtering results with a $32 \times 32$ window would have been much worse in terms of noise reduction and residues removal, and hence, the comparison with the rest of the methods would have not been reliable. 
TABLE II

Filter PARAMETERS

\begin{tabular}{lccc}
\hline \multicolumn{1}{c}{$\begin{array}{c}\text { Filter } \\
\text { type }\end{array}$} & $\begin{array}{c}\text { Window } \\
\text { size }\end{array}$ & $\begin{array}{c}\text { Coherence } \\
\text { estimation }\end{array}$ & $\begin{array}{c}\text { Filter } \\
\text { parameter }\end{array}$ \\
\hline Goldstein & $256 \times 256$ & - & $\alpha=0.9$ \\
Modified & $256 \times 256$ & $5 \times 5$ & Adaptive \\
Goldstein & Adaptive & $5 \times 5$ & Adaptive \\
Pixel-by-pixel & $256 \times 256$ to $8 \times 8$ & $5 \times 5$ & Adaptive \\
RASF & $256 \times 256$ to $8 \times 8$ & $5 \times 5$ & Adaptive \\
Our method & & & \\
\hline
\end{tabular}

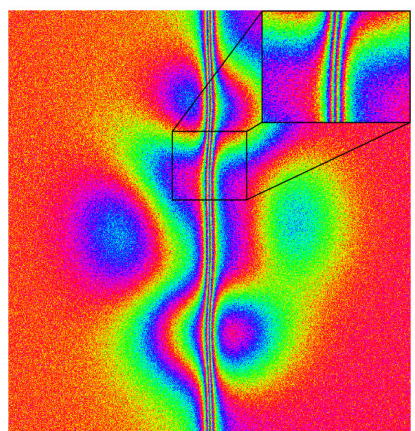

(a)

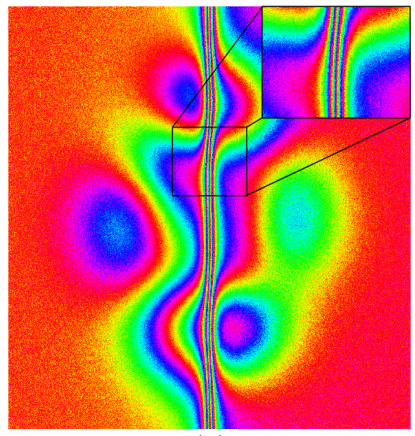

(c)

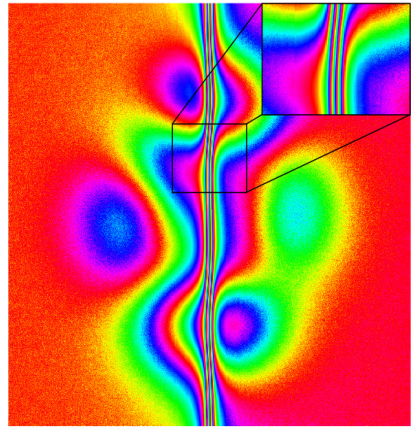

(e)

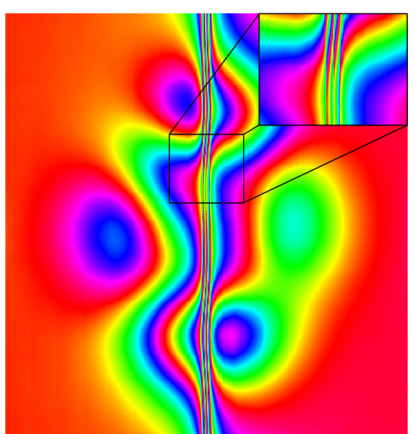

(b)

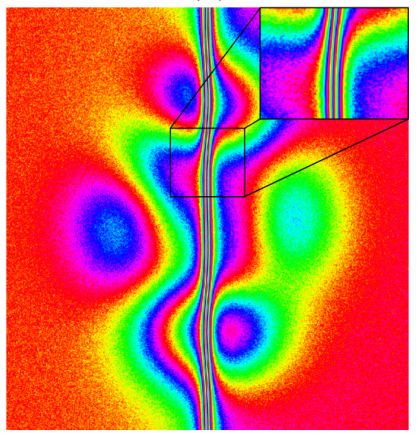

(d)

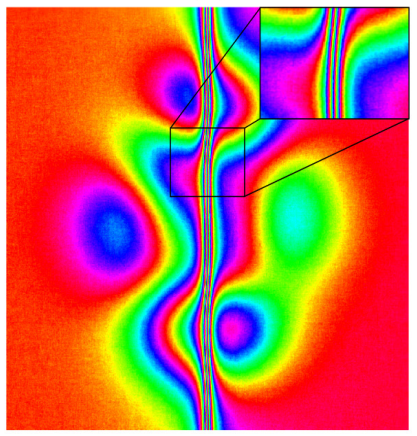

(f)
Fig. 5. Filtering results of interferogram $I_{1}$ with different methods. (a) Original noisy phase. (b) Goldstein filter. (c) Adaptive Goldstein filter. (d) Pixel-by-pixel filter. (e) RASF. (f) Proposed method.

In Fig. 5, we show the filtering results of the highest quality interferogram $I_{1}$. All the filters show good results in this case, and the noise is globally suppressed. Also, fringe continuity is preserved with all the adaptive methods but not with the standard Goldstein filter, where clearly overfiltering effects are visible in the dense fringe area of the central part of the interferogram. This is due to the large value of the filtering parameter $\alpha(0.9)$ for which noise reduction is almost total. In this regard, the Goldstein-filtered phase is almost identical to the true phase in wide areas with low- or null-frequency ramps, for instance, in the bottom-right corner of Fig. 5(b), as the filter is strong despite the phase quality is already high. Consequently, in this case, the standard Goldstein method offers the best result in noise reduction, but some fringes are lost. In Table III, we show the filtering results according to the quality criteria previously mentioned. We can see that the few residues in the original phase are reduced to 0 in all the cases. Among them, our method shows the best global result in terms of MSE.

The results of filtering the interferogram $I_{2}$ are shown in Fig. 6, and summarized in Table III. As it can be observed in Fig. 6(a), the noise level is clearly higher than in the previous simulation, and the number of residues is also larger. Although noise is almost completely suppressed, the Goldstein-filtered phase is still overfiltered in the highdensity fringe area. Concerning the adaptive Goldstein and the pixel-by-pixel filters, they both show good results, especially in fringe preservation. However, noise reduction is not as effective as with the other filters, and the final number of residues is larger in comparison with the other methods. The RASF filter shows a good performance in noise reduction and the improvement in terms of remaining residues is almost total. However, in this case, the resulting phase is overfiltered in the high-gradient area, and some detailed features are lost. Then, according to these two simulations, we can deduce that our proposed method offers a larger improvement if the phase gradient is high or the noise is high, proving that it achieves the best balance between noise suppression and fringe preservation in comparison with the rest of the methods.

The third simulation corresponds to interferogram $I_{3}$, whose original phase can be observed in Fig. 7(a). In this case, the noise level is considerably higher than before and the original number of residues is very large, as shown in Table III. Then, completely, noise reduction cannot be achieved without the use of large filtering windows. In addition, the estimation of the local phase ramp inside the window becomes a very challenging task, as even pure noise may present a dominant component in its Fourier transform. Consequently, noise reduction along with fringe preservation is nearly unattainable.

As it can be observed in Fig. 7(b) and (c), both the standard Goldstein and its adaptive version present almost the same performance as a result of the low-coherence values. Then, the filtering parameter $\alpha$ is very large, making the filtering strong. The number of residues is also similar to these two filters.

Concerning the pixel-by-pixel filter, the size of the individual filtering windows is not enough to completely suppress the noise with just a single filtering operation, as shown in Fig. 7(d). The number of residues of the filtered interferogram is the largest among all the filtering methods and so does the MSE.

The RASF filter and the proposed method show the best results both in residues' suppression and MSE. In fact, the improvement in terms of residues is almost identical, being our method just $0.01 \%$ better. However, as this corresponds 


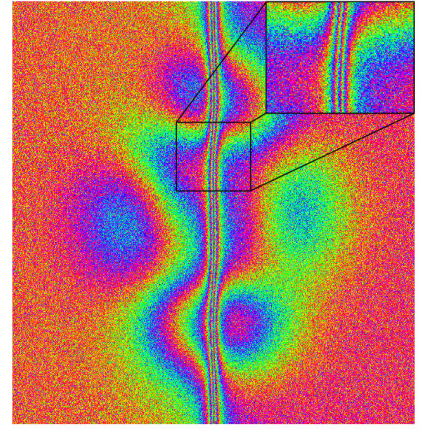

(a)

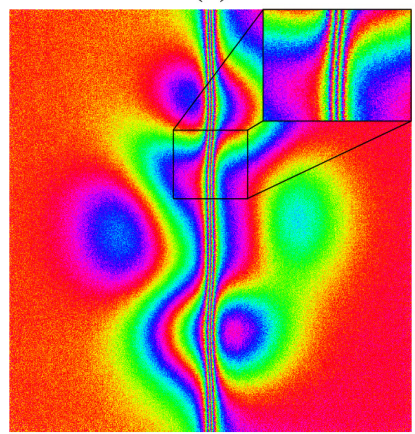

(c)

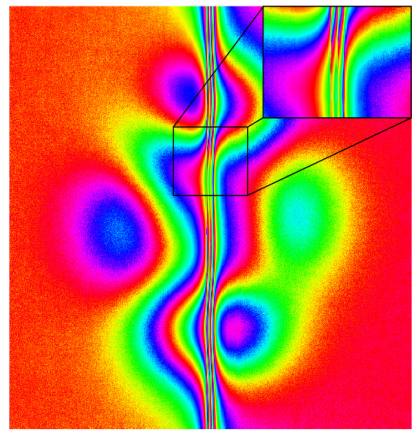

(e)

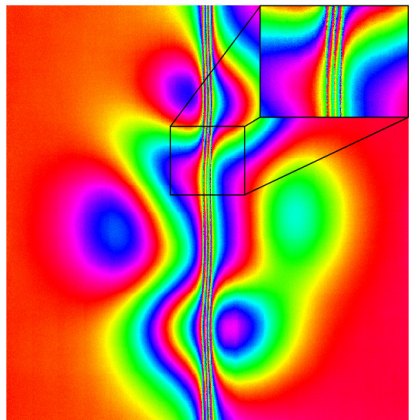

(b)

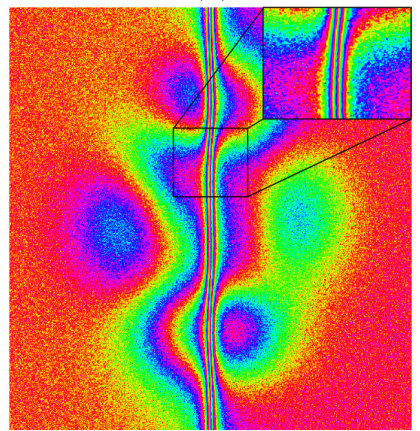

(d)

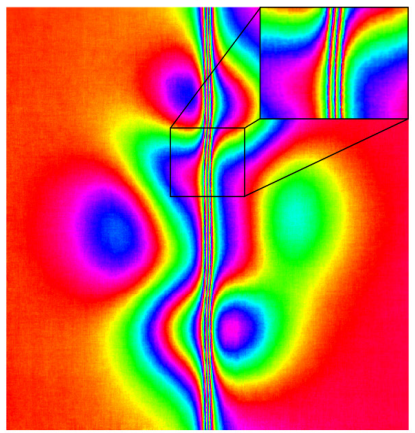

(f)

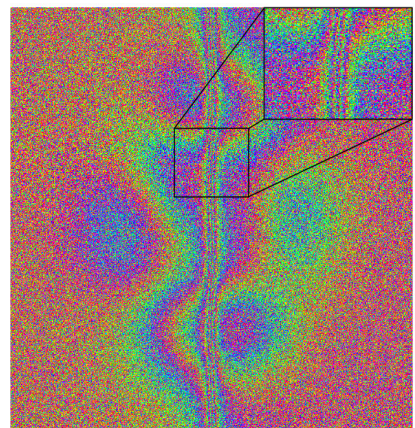

(a)

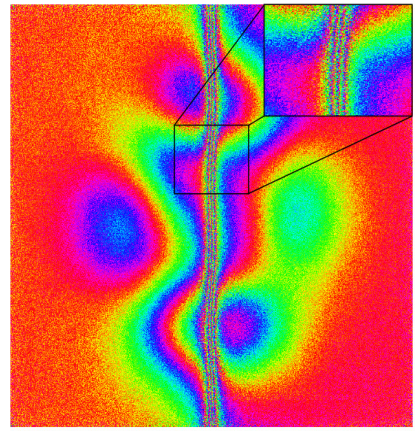

(c)

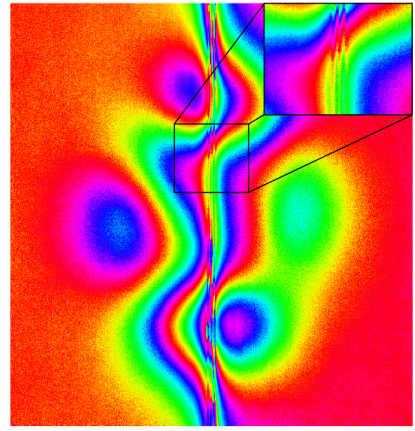

(e)

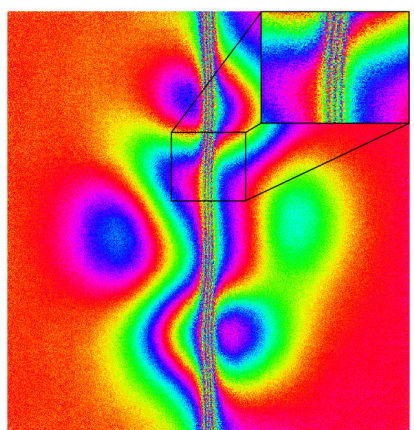

(b)

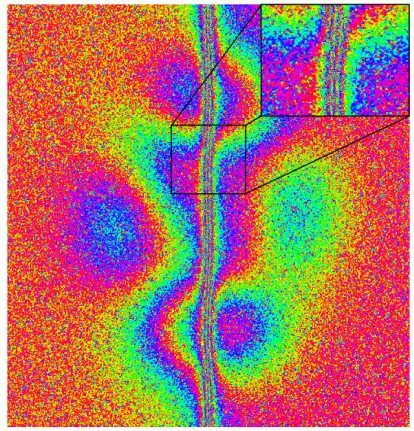

(d)

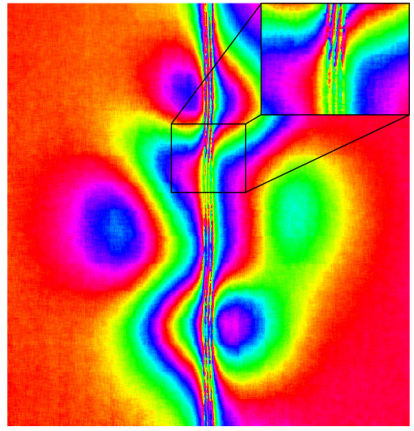

(f)

Fig. 6. Filtering results of interferogram $I_{2}$ with different methods. (a) Original noisy phase. (b) Goldstein filter. (c) Adaptive Goldstein filter. (d) Pixel-by-pixel filter. (e) RASF. (f) Proposed method.

to the mean improvement after 100 realizations and the number of samples (pixels) is large enough, a nonparametric statistical test proves that the improvement is significant. More specifically, in this simulation, a $p$-value equal to $9.731 \times 10^{-6}$ is obtained. This value is below the statistical significance of 0.01 which is usually employed.

The overfiltering can be limited with the use of smaller filtering windows in the initial steps of the algorithm. In Fig. 8, we show the filtered phases of the simulated interferogram $I_{3}$ with our method when different initial window sizes have been employed. As expected, noise suppression becomes less effective with smaller windows, but the phase is less likely to be overfiltered. For instance, when an initial window size of $32 \times 32$ is used, the detailed features are correctly preserved, but noise is less reduced in low-frequency areas. Nevertheless, noise reduction is still very effective with a $32 \times 32$ initial filtering window and the improvement is quite remarkable, as shown in Fig. 8(c).

Fig. 7. Filtering results of interferogram $I_{3}$ with different methods. (a) Original noisy phase. (b) Goldstein filter. (c) Adaptive Goldstein filter. (d) Pixel-by-pixel filter. (e) RASF. (f) Proposed method.

The last simulation corresponds to an extremely noisy interferogram. The standard deviation of the noise is out of the limit derived in [16] concerning the phase statistics and represented in Fig. 1 (which is around $1.8 \mathrm{rad}$ ). The phase standard deviation has been manually fixed to $2.569 \mathrm{rad}$ in order to add noise to the data. All the filtering results are represented in Fig. 9. Apparently, the original phase seems to be pure noise so that the phase structure is completely masked by the noise, as shown in Fig. 9(a). As in the previous simulation, the Goldstein filter and the modified Goldstein filters offer the same results, as the coherence values are close to 0 , making the filter parameter $\alpha$ very large. However, both filters do not recover useful measurements (even with a $256 \times 256$ filtering window), and the original and filtered phases seem to be the same. Concerning the pixel-by-pixel filter, the maximum window size has been used to filter each pixel in the image. Noise reduction is almost negligible with this filter. However, as it can be seen in Fig. 9(e) and (f), 


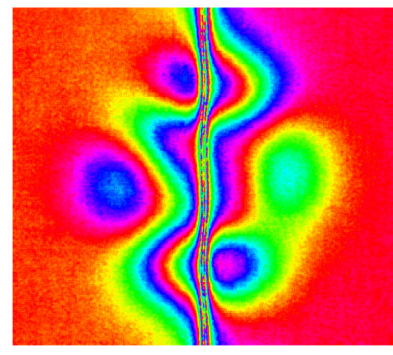

(a)

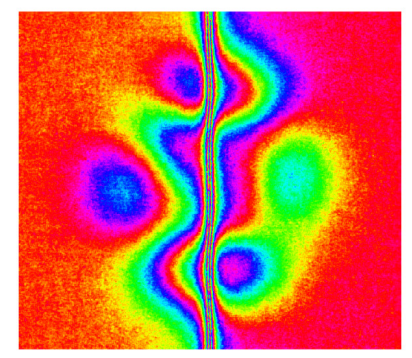

(b)

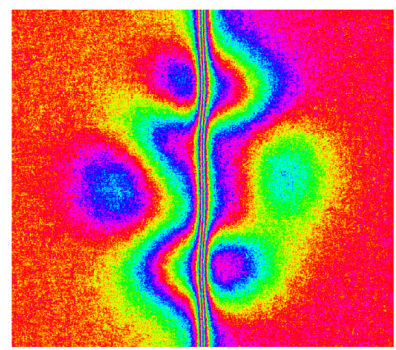

(c)

Fig. 8. Filtering results of interferogram $I_{3}$ with our proposed method with different initial windows sizes. (a) $128 \times 128$. (b) $64 \times 64$. (c) $32 \times 32$.

both iterative filters are able to greatly suppress the noise and, at least, to recover some parts of the original signal. Moreover, our proposed method shows a better performance in the spatial smoothness of the phase values, whereas the RASF filter presents some artifacts and more discontinuities in the filtered phase. Obviously, the recovering of the detailed fringes is almost infeasible with such a degraded original interferogram.

The filtering results for this interferogram are summarized in Table III. As expected, only the iterative methods present a major improvement in terms of the final number of residues. It can be observed that the proposed filter offers again the best result in terms of residues and MSE. Instead, the improvement is much less significant with the other three filters.

From this last simulation, a potential problem of the proposed method can be predicted. As previously mentioned, the estimation of a reliable local phase ramp is a very difficult task in extremely noisy areas, considering that even a fully uncorrelated interferogram patch (pure noise) has a dominant component in the frequency domain. Consequently, wrong phase ramps could be estimated and readded at the filtering step (Fig. 2), so that some nongenuine interferometric fringes may be present in the filter's output. According to this situation, an analysis of the coherence and the phase standard deviation can be considered to derive a minimum threshold from which the filter should proceed. Below this quality threshold, the filter should not run, and its output should be the input noisy phase. In this case, the user would know that there is no useful signal to be recovered. However, a deep analysis of this situation should be carried out as it is not an evident task and it would suppose an improvement of the proposed method for such extreme cases.

Finally, in order to verify the effectiveness of the proposed kernel against others, we filter interferogram $I_{3}$ with our

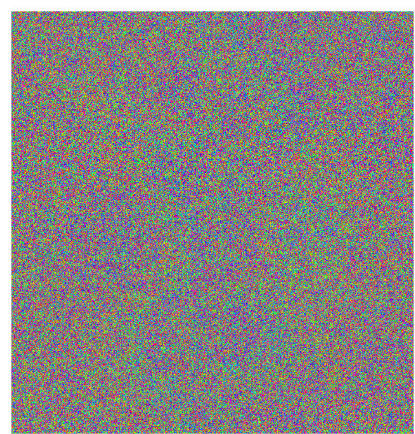

(a)

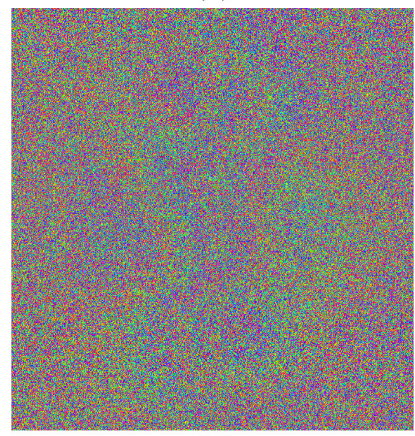

(c)

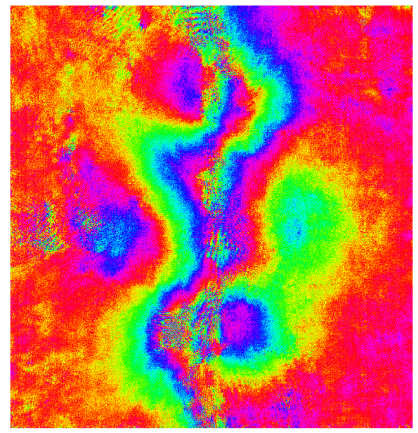

(e)

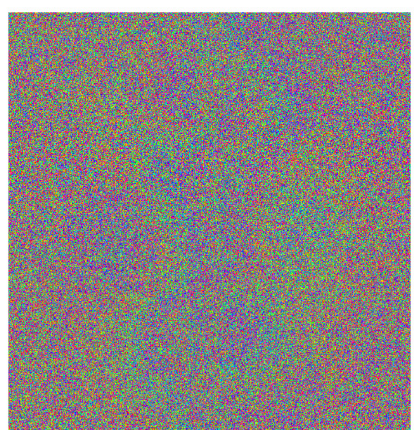

(b)

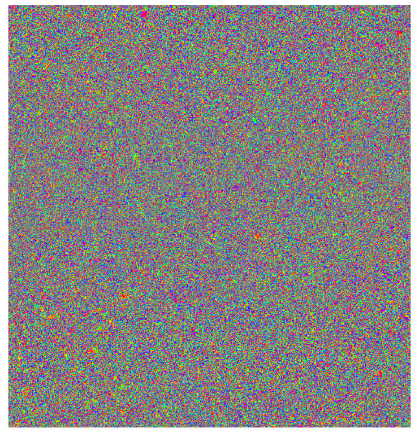

(d)

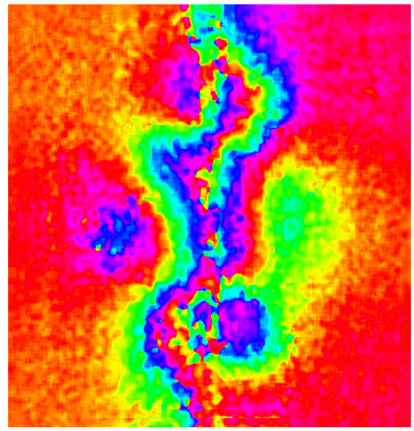

(f)
Fig. 9. Filtering results of interferogram $I_{4}$ with different methods. (a) Original noisy phase. (b) Goldstein filter. (c) Adaptive Goldstein filter (d) Pixel-by-pixel filter. (e) RASF. (f) Proposed method.

method and two different convolution kernels. To provide a better comparison, a single iteration with a $32 \times 32$ is applied. According to our formulation, the size of the filtering kernel is $6 \times 6$ pixels. The Chebyshev-polynomial-based kernel is compared with a Gaussian and a mean kernel of the same size. The resulting phases are represented in Fig. 10, and the quantitative filtering results are shown in Table IV. As it can be observed, the proposed kernel provides better filtering results: the number of residues is much lower and so does the MSE value.

\section{B. Real Interferogram: RADARSAT-2 Data Over Mount Etna Volcanic Eruption}

In this section, we show the filtering results of a real SAR differential interferogram corresponding to the Mount Etna's volcanic eruption in May 2008. More specifically, the interferogram is the result of the combination of two images acquired in dates $2008-05-05$ and 2008-05-29 by the 
TABLE III

Performance Analysis of Different Phase Filters FOR EACH SIMULATED INTERFEROGRAM

\begin{tabular}{lccc}
\hline & Residue number & Improvement & MSE \\
\hline Interferogram $I_{1}$ & & & \\
\hline Original & 86 & - & 0.264 \\
Goldstein & 0 & $100 \%$ & 0.019 \\
Adaptive Goldstein & 0 & $100 \%$ & 0.081 \\
Pixel-by-pixel & 0 & $100 \%$ & 0.063 \\
RASF & 0 & $100 \%$ & 0.031 \\
Our method & $\mathbf{0}$ & $\mathbf{1 0 0 \%}$ & $\mathbf{0 . 0 1 1}$ \\
\hline \hline & & & \\
Interferogram $I_{2}$ & & & \\
\hline Original & 66391 & - & 0.941 \\
Goldstein & 164 & $99.75 \%$ & 0.022 \\
Adaptive Goldstein & 442 & $99.33 \%$ & 0.091 \\
Pixel-by-pixel & 2627 & $96.04 \%$ & 0.253 \\
RASF & 12 & $99.98 \%$ & 0.041 \\
Our method & $\mathbf{0}$ & $\mathbf{1 0 0 \%}$ & $\mathbf{0 . 0 1 9}$ \\
\hline \hline
\end{tabular}

Interferogram $I_{3}$

\begin{tabular}{lccc}
\hline Original & 264907 & - & 2.135 \\
Goldstein & 6600 & $97.51 \%$ & 0.117 \\
Adaptive Goldstein & 8519 & $96.78 \%$ & 0.228 \\
Pixel-by-pixel & 52034 & $80.36 \%$ & 1.043 \\
RASF & 95 & $99.96 \%$ & 0.094 \\
Our method & $\mathbf{7 1}$ & $\mathbf{9 9 . 9 7 \%}$ & $\mathbf{0 . 0 8 0}$ \\
\hline \hline Interferogram $I_{4}$ & & & \\
\hline Original & 331415 & - & 3.145 \\
Goldstein & 328696 & $0.82 \%$ & 3.068 \\
Adaptive Goldstein & 328654 & $0.83 \%$ & 3.069 \\
Pixel-by-pixel & 269945 & $18.55 \%$ & 3.147 \\
RASF & 4293 & $98.71 \%$ & 0.523 \\
Our method & $\mathbf{8 6 3}$ & $\mathbf{9 9 . 7 3 \%}$ & $\mathbf{0 . 4 0 1}$ \\
\hline
\end{tabular}

TABLE IV

Performance Analysis of Our Proposed Method With THREE DiFFERENT FILTERING KERNELS

\begin{tabular}{lccc}
\hline Interferogram $I_{3}$ & Residue number & Improvement & MSE \\
\hline Original & 264907 & - & 2.135 \\
$\begin{array}{l}\text { Our method with } \\
\text { a } 6 \times 6 \text { Gaussian kernel }\end{array}$ & 20122 & $92.40 \%$ & 0.493 \\
$\begin{array}{l}\text { Our method with } \\
\text { a } 6 \times 6 \text { mean kernel }\end{array}$ & 7648 & $97.11 \%$ & 0.306 \\
$\begin{array}{l}\text { Our method with } \\
\text { a 6 } \times \text { 6 Chebyshev kernel }\end{array}$ & $\mathbf{1 3 5 5}$ & $\mathbf{9 9 . 4 9 \%}$ & $\mathbf{0 . 1 7 8}$ \\
\hline
\end{tabular}

RADARSAT-2 satellite in the beam mode FQ29. The nearrange and far-range incidence angles are $46.8^{\circ}$ and $48.0^{\circ}$, respectively. All the filters have been evaluated with fullresolution (single-look complex) input data, and the polarimetric channel is $\mathrm{HH}$. The size of the processed interferogram is 3700 pixels (range dimension) $\times 6000$ pixels (azimuth dimension). Note that the sea area in the right part of the scene has been masked out and has not been filtered. All the filtering

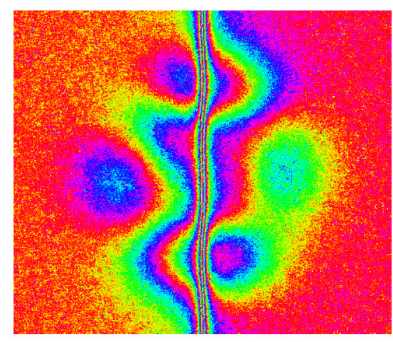

(a)

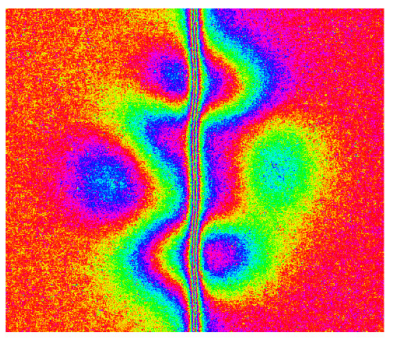

(b)

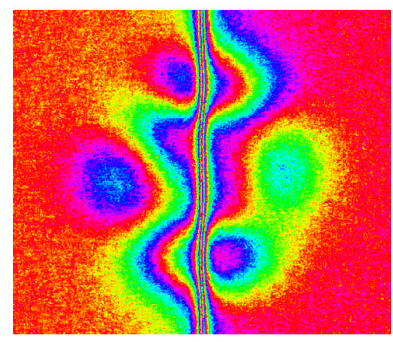

(c)

Fig. 10. Filtering results of interferogram $I_{3}$ in a single iteration with three different kernels. (a) Gaussian kernel. (b) Mean kernel. (c) Chebyshevpolynomial-based kernel.

TABLE V

Performance Analysis of Different Phase FILTERS FOR RADARSAT-2 DATA SET

\begin{tabular}{lcc}
\hline Interferogram & Residue number & Improvement \\
\hline Original & 4353927 & - \\
Goldstein & 644005 & $85.21 \%$ \\
Adaptive Goldstein & 1140388 & $73.81 \%$ \\
Pixel-by-pixel & 1738890 & $60.06 \%$ \\
RASF & 31097 & $99.29 \%$ \\
Our method & $\mathbf{3 5 0 0}$ & $\mathbf{9 9 . 9 2 \%}$ \\
\hline
\end{tabular}

parameters are the same as the ones of the simulations of Section IV-A (they are summarized in Table II). The resulting differential phases are shown in Fig. 11. As it can be seen in Fig. 11(a), the scene presents a high level of noise, which can be mainly due to temporal decorrelation (both images are acquired at a revisit time of 24 days) and due to presence of vegetation. The evaluation of the filtering results is shown in Table V.

As expected, the number of residues of the original phase is very large. A visual inspection of Fig. 11(d) demonstrates that the noise reduction with the pixel-by-pixel filtering strategy is limited in very noisy areas. This can also be deduced from the large number of residues that the filtered phase still has. Concerning the Goldstein filter and the coherenceadaptive Goldstein filter, whose filtered phases are shown in Fig. 11(b) and (c), the improvement in noise reduction is noticeable, but the remaining number of residues is also high. Consequently, the phase unwrapping step would be difficult. From Fig. 9(e) and (f), we can see that noise can only be significantly suppressed by filtering the original interferogram multiple times as it is proposed in the RASF filter and with our method. The RASF filter shows a good improvement in terms 


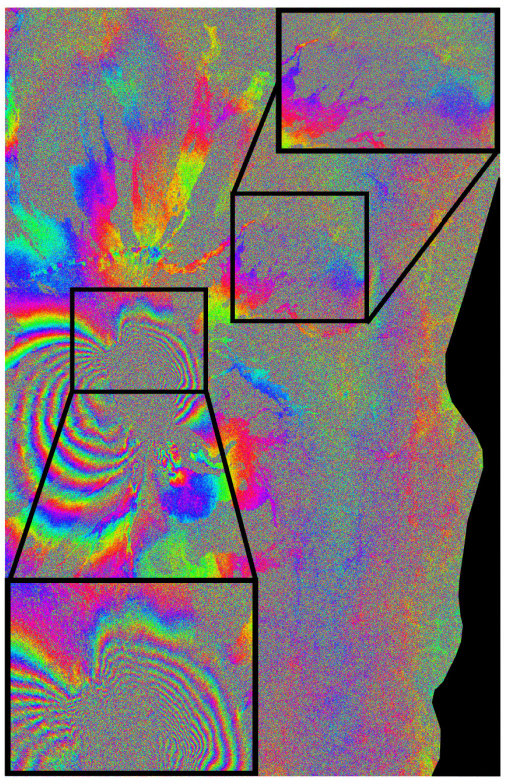

(a)

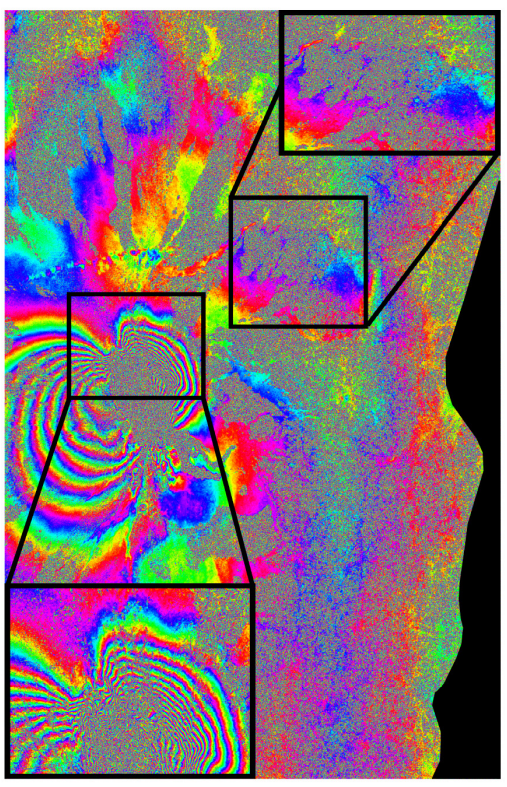

(d)

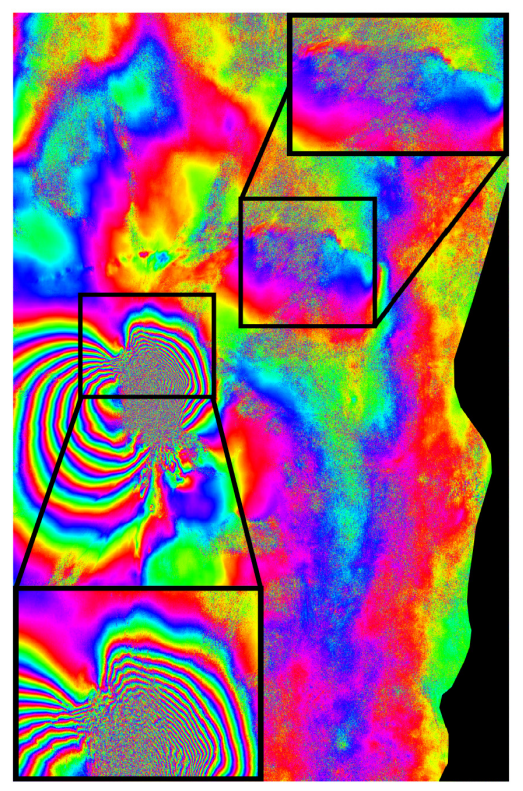

(b)

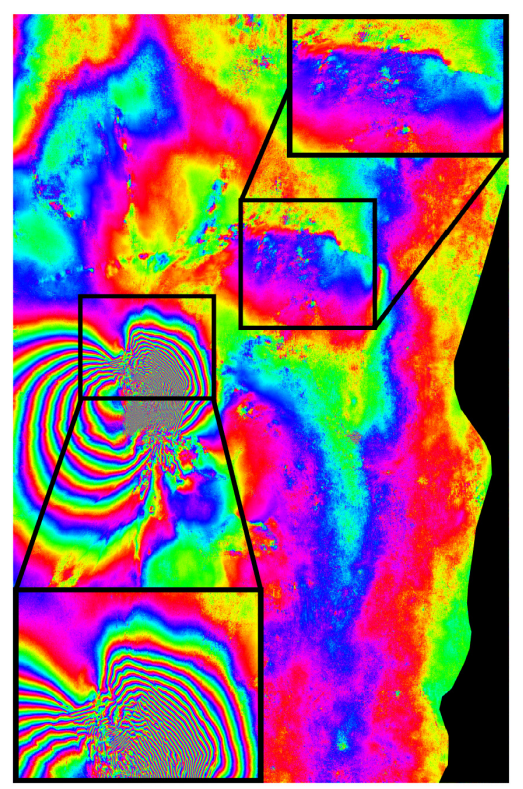

(e)

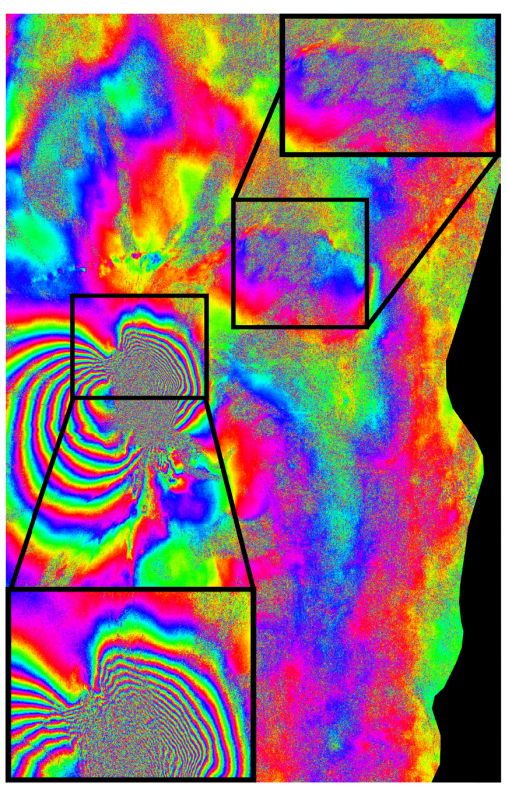

(c)

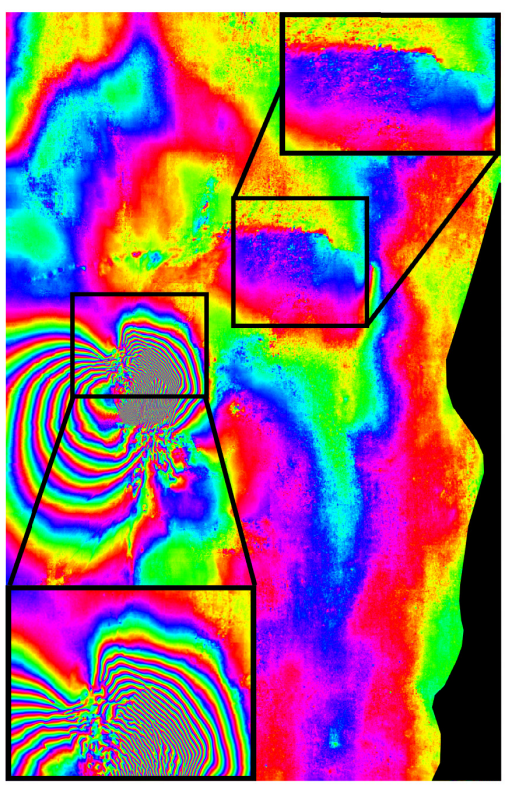

(f)

Fig. 11. RADARSAT-2 interferogram over the Mount Etna's volcanic eruption filtered with different methods. (a) Original noisy phase. (b) Goldstein filter. (c) Adaptive Goldstein filter. (d) Pixel-by-pixel filter. (e) RASF. (f) Proposed method.

of noise suppression, and the number of residues is greatly reduced.

For a detailed comparison, we have extracted two different areas of the interferogram. One of them corresponds to a high deformation area caused by the volcanic eruption, where strong phase gradients are present. The other region corresponds to a highly decorrelated zone. As it can observed in the bottom of Fig. 11, the details of the deformation are globally well preserved by all filtering strategies. However, only the two iterative methods assure a significantly cleaner phase while preserving the strong gradients caused by the deformation. Moreover, comparing Fig. 9(e) and (f), the filtered phase around the volcano crater with our method exhibits smoother and more continuous values than the RASF-filtered phase. This is more noticeable if we compare the filtering results on the noisy area displayed in the top of Fig. 11. The resulting phase with our filter presents less discontinuities, whereas the RASFfiltered phase is noisier and shows some artifacts. Finally, regarding the quantitative results of each filter (summarized in Table V), it is proven that our proposed method has the best performance in terms of residues' suppression. Consequently, it is also proved that the proposed filter modifications improve the quality of the final phase with respect to the RASF. 


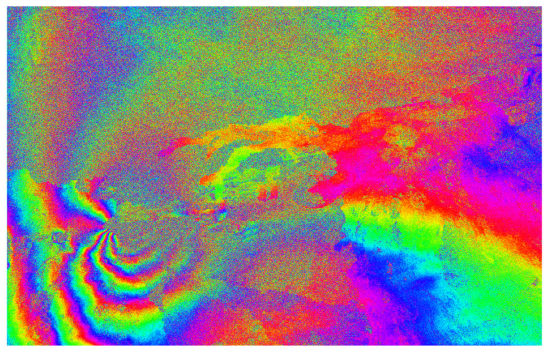

(a)

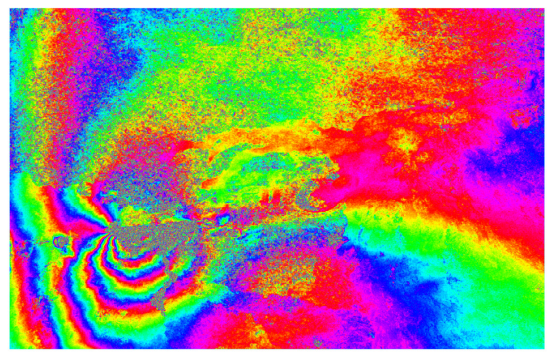

(d)

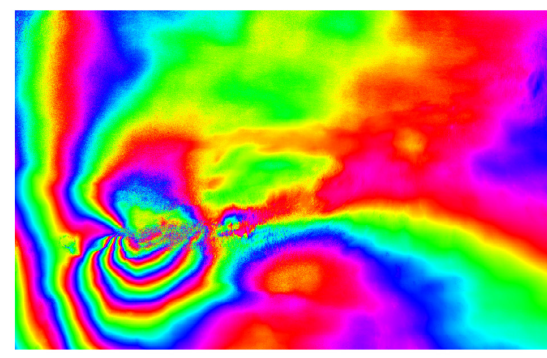

(b)

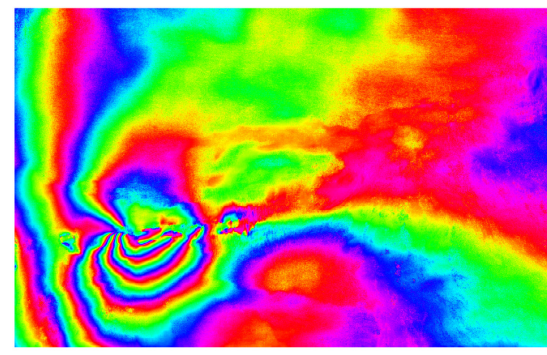

(e)

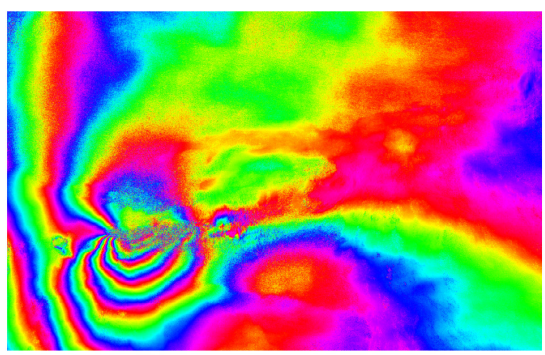

(c)

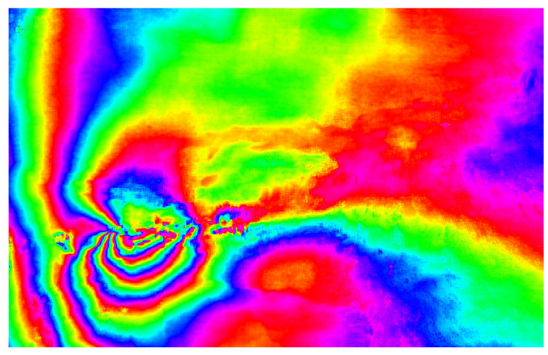

(f)

Fig. 12. UAVSAR interferogram over the Kilauea volcanic eruption filtered with different methods. (a) Original noisy phase. (b) Goldstein filter. (c) Adaptive Goldstein filter. (d) Pixel-by-pixel filter. (e) RASF. (f) Proposed method.

TABLE VI

PERFormance ANALysis of DifFEREnt Phase FiLters FOR UAVSAR DATA SET

\begin{tabular}{lcc}
\hline Interferogram & Residue number & Improvement \\
\hline Original & 415409 & - \\
Goldstein & 5462 & $98.69 \%$ \\
Adaptive Goldstein & 11509 & $97.23 \%$ \\
Pixel-by-pixel & 100635 & $75.78 \%$ \\
RASF & 172 & $99.96 \%$ \\
Our method & $\mathbf{8 0}$ & $\mathbf{9 9 . 9 8 \%}$ \\
\hline
\end{tabular}

\section{Real Interferogram: UAVSAR Data Over Kilauea Volcanic Eruption}

The second real data set corresponds to a UAVSAR interferogram mapping the Kilauea volcanic eruption in March 2011. Images were acquired in dates 2010-01-06 and 2011-03-05. The near- and far-range incidence angles are $21.9^{\circ}$ and $65.7^{\circ}$, respectively. The processed image size is $2000 \times 1500$ pixels (range $\times$ azimuth) and the interferograms correspond to the HH polarimetric channel. Filtering results are shown in Fig. 12.

All the filtering parameters are the same as in the previous examples except for the dimensions of the window employed to estimate the coherence, whose size is $3 \times 12$ pixels (range $\times$ azimuth). These dimensions correspond to the number of looks used to compute the coherence map provided by UAVSAR. The improvement in terms of the final residue number is summarized in Table VI. All filters show a good performance in noise suppression and fringe preservation. Note that the quality of the original data is better than in the Etna case. The pixel-by-pixel filter correctly preserves the majority of the details of deformation, but noise suppression is clearly not enough in the most noisy areas. For the rest of the filters, the improvement in terms of residues is remarkable. In fact, more than $97 \%$ of the residues are eliminated and the interferometric phase fringes corresponding to the deformation are well preserved. Finally, our proposed method still shows the best results in terms of residues' removal.

\section{CONClusion}

We have presented an improved interferometric phase filter that is based on the conventional Goldstein filter formulation in combination with an iterative methodology. The proposed filter introduces some modifications with respect to another known iterative approach, called RASF, that have shown to improve the quality of the filtering. The use of adaptive Chebyshev-polynomial-based kernels as the smoothing operator has proved to effectively suppress phase noise. Furthermore, the adaptive windowing in each filtering iteration has shown to progressively denoise the original phase, while the interferometric fringes corresponding to the deformation pattern are preserved. Additionally, the proposed method is able to recover useful measurements even with extremely noisy data.

The new method has been compared with four already existing filters. For comparison purposes, we have employed the same filtering parameters for all the filters (for instance, the same window size in block-filtering methods). Also, we have tried to maximize the filtering effectiveness of each filter, e.g., by fixing a large $\alpha$ value in the conventional Goldstein filter and using large filtering windows. It has been shown that our proposed method offers the best filtering results, both with simulated interferograms and real SAR data from different sensors. In all the evaluated cases, the number of residues is greatly reduced (almost to $100 \%$ ). It has been 
proven that the proposed method offers a larger improvement if strong phase gradients are present or when noise is very high, showing that the main objectives of the filter, i.e. noise suppression in combination with fringe continuity preservation, have been achieved. Finally, as a future work, we plan to improve the proposed method by considering extreme noise levels and including a minimum quality threshold from which the filter should proceed, hence avoiding wrong phase ramps estimates or phase artifacts that could be present in the filtered signal if some areas are fully uncorrelated.

\section{ACKNOWLEDGMENT}

RADARSAT-2 Data and Products MacDonald, Dettwiler and Associates Ltd. (MDA) (2008)—All Rights Reserved. RADARSAT is an official trademark of the Canadian Space Agency (CSA). All RADARSAT-2 images were provided by MDA and CSA in the framework of project SOAR 5087. The UAVSAR differential interferogram and the associated coherence map have been provided by NASA-Jet Propulsion Laboratory.

\section{REFERENCES}

[1] D. Massonnet et al., "The displacement field of the Landers earthquake mapped by radar interferometry," Nature, vol. 364, no. 6433, pp. 138-142, 1993

[2] R. Bürgmann, P. A. Rosen, and E. J. Fielding, "Synthetic aperture radar interferometry to measure Earth's surface topography and its deformation," Апnи. Rev. Earth Planetary Sci., vol. 28, no. 1, pp. 169-209, 2000.

[3] A. Ferretti, C. Prati, and F. Rocca, "Permanent scatterers in SAR interferometry," IEEE Trans. Geosci. Remote Sens., vol. 39, no. 1, pp. 8-20, Jan. 2001.

[4] P. Berardino, G. Fornaro, R. Lanari, and E. Sansosti, "A new algorithm for surface deformation monitoring based on small baseline differential SAR interferograms," IEEE Trans. Geosci. Remote Sens., vol. 40, no. 11, pp. 2375-2383, Nov. 2002.

[5] A. Hooper, H. Zebker, P. Segall, and B. Kampes, "A new method for measuring deformation on volcanoes and other natural terrains using InSAR persistent scatterers," Geophys. Res. Lett., vol. 31, no. 23 , p. 123611, 2004. [Online]. Available: http://dx.doi.org/10.1029/ 2004GL021737

[6] H. A. Zebker and J. Villasenor, "Decorrelation in interferometric radar echoes," IEEE Trans. Geosci. Remote Sens., vol. 30, no. 5, pp. 950-959, Sep. 1992.

[7] R. M. Goldstein, H. A. Zebker, and C. L. Werner, "Satellite radar interferometry: Two-dimensional phase unwrapping," Radio Sci., vol. 23, no. 4, pp. 713-720, Aug. 1988.

[8] D. C. Ghiglia and M. D. Pritt, Two-Dimensional Phase Unwrapping: Theory, Algorithms, and Software. Hoboken, NJ, USA: Wiley, May 1998.

[9] R. M. Goldstein and C. L. Werner, "Radar interferogram filtering for geophysical applications," Geophys. Res. Lett., vol. 25, no. 21, pp. 4035-4038, Nov. 1998. [Online]. Available: http://dx.doi.org/ 10.1029/1998GL900033

[10] J. McClellan, "Artifacts in alpha-rooting of images," in Proc. IEEE Int. Conf. Acoust., Speech, Signal Process. (ICASSP), vol. 5. Apr. 1980, pp. $449-452$.

[11] I. Baran, M. P. Stewart, B. M. Kampes, Z. Perski, and P. Lilly, "A modification to the Goldstein radar interferogram filter," IEEE Trans. Geosci. Remote Sens., vol. 41, no. 9, pp. 2114-2118, Sep. 2003.

[12] Z. Suo, J. Zhang, M. Li, Q. Zhang, and C. Fang, "Improved InSAR phase noise filter in frequency domain," IEEE Trans. Geosci. Remote Sens., vol. 54, no. 2, pp. 1185-1195, Feb. 2016.

[13] P. J. González, "A recursive adaptive spectral interferogram phase filtering method," in Proc. Wegener Symp., Leeds, U.K., Sep. 2014.

[14] P. J. González, "RSF: A robust non-parametric phase filtering method for automatic processing of Sentinel-1 interferograms," in Proc. Adv. Sci. Appl. SAR Interferometry Sentinel-1 InSAR Workshop (FRINGE), Frascati, Italy, Mar. 2015. [Online]. Available: http://seom.esa.int/fringe2015/page_session13.php\#83p
[15] H. A. Zebker and K. Chen, "Accurate estimation of correlation in InSAR observations," IEEE Geosci. Remote Sens. Lett., vol. 2, no. 2 pp. 124-127, Apr. 2005.

[16] J.-S. Lee, K. W. Hoppel, S. A. Mango, and A. R. Miller, "Intensity and phase statistics of multilook polarimetric and interferometric SAR imagery," IEEE Trans. Geosci. Remote Sens., vol. 32, no. 5 pp. 1017-1028, Sep. 1994

[17] J. C. Mason and D. C. Handscomb, Chebyshev Polynomials. Boca Raton, FL, USA: CRC Press, 2003.

[18] E. Trouvé, M. Caramma, and H. Maître, "Fringe detection in noisy complex interferograms," Appl. Opt., vol. 35, no. 20, pp. 3799-3806, Jul. 1996. [Online]. Available: http://ao.osa.org/abstract.cfm?URI=ao35-20-3799

[19] Z. Y. Suo, Z. F. Li, and Z. Bao, "A new strategy to estimate local fringe frequencies for InSAR phase noise reduction," IEEE Geosci. Remote Sens. Lett., vol. 7, no. 4, pp. 771-775, Oct. 2010.

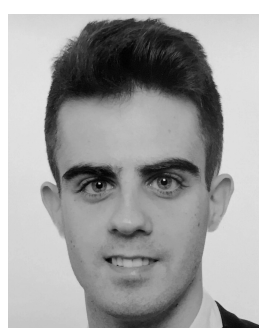

Alejandro Mestre-Quereda (S'18) was born in Madrid, Spain, in 1990. He received the B.S. degree in telecommunication engineering and the M.Sc. degree in telecommunication engineering from the University of Alicante, Alicante, Spain, in 2015 and 2017, respectively, where he is currently pursuing the $\mathrm{Ph} . \mathrm{D}$. degree.

Since 2016, he has been a Pre-Doctoral Fellow with the Signals, Systems and Telecomunnication Group, University of Alicante. His research interests include differential synthetic aperture radar interferometry (DInSAR) and polarimetric DInSAR processing methods for monitoring and characterizing geophysical hazards with orbital SAR data.

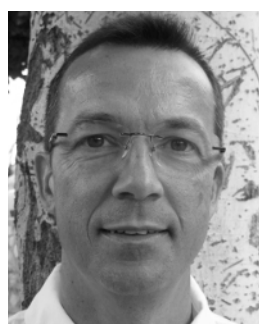

Juan M. Lopez-Sanchez (S'94-M'00-SM'05) was born in Alicante, Spain, in 1972. He received the Ingeniero (M.S). and Doctor Ingeniero (Ph.D.) degrees in telecommunication engineering from the Technical University of Valencia, Valencia, Spain, in 1996 and 2000, respectively.

From 1998 to 1999, he was a Pre-Doctoral Grant Holder with the Space Applications Institute, Joint Research Centre of the European Commission, Ispra, Italy. Since 2000, he has been the Leader of the Signals, Systems and Telecommunication Group, University of Alicante, Alicante, where he has also been a Full Professor since 2011. He has co-authored more than 60 papers in refereed journals and more than 110 papers and presentations in international conferences and symposia. His research interests include microwave remote sensing for inversion of biophysical parameters, polarimetric and interferometric techniques, synthetic aperture radar imaging algorithms, and applications of radar remote sensing in agriculture and geophysics.

Dr. Lopez-Sanchez received the Indra Award for the best Ph.D. thesis about radar in Spain in 2001. From 2006 to 2012, he was the Chair of the Spanish Chapter of the IEEE Geoscience and Remote Sensing Society. 


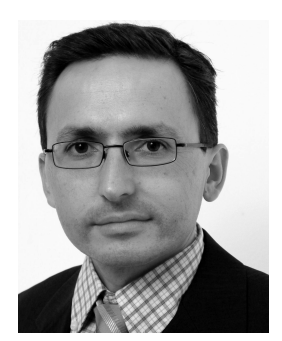

Jesus Selva received the M.Sc. degree in communications engineering from the Polytechnic University of Valencia (UPV), Valencia, Spain, in 1994, the M.Sc. degree in mathematics from the National Distance Education University, Madrid, Spain, in 2000, and the Ph.D. degree in communications engineering from the Polytechnic University of Catalonia, Barcelona, Spain, in 2004.

In 1995, he was a grant holder at UPV. From 1996 to 1998, he was a Trainee with the Spanish Ministry of Education, European Space Agency, Frascati, Italy, and Noordwijk, The Netherlands. From 1998 to 2004, he was Researcher with the German Aerospace Centre, Wessling, Germany. From 2004 to 2011, he was the Juan de la Cierva and Ramon y Cajal Research Fellow with the University of Alicante, Alicante, Spain, where he has been an Associate Professor since 2012. His research interests include interpolation for signal processing, sampling theory, navigation systems, synchronization circuits, array processing, and estimation theory.

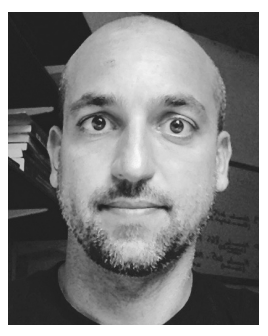

Pablo J. Gonzalez received the M.Sc. degree in geological sciences from the University of Granada, Granada, Spain, in 2003, and the M.Sc. (DEA) degree in geodetic and cartographic engineering and the $\mathrm{Ph} . \mathrm{D}$. degree in geodesy from the Universidad Complutense de Madrid, Madrid, Spain, in 2006 and 2010, respectively.

He was a Post-Doctoral Research Fellow with the University of Leeds, Leeds, U.K., and the Centre for the Observation and Modelling of Earthquakes, Volcanoes and Tectonics, Natural Environmental Research Council, and a Research Associate and a Banting Fellow with The University of Western Ontario, London, ON, Canada. Since 2016, he has been with the University of Liverpool, Liverpool, U.K., where he is currently a Lecturer in geophysics. He has authored almost 50 papers in international peer-reviewed journals. His research interests include the innovative application of space geodesy, and more recently high-resolution topography data, to understand the mechanisms that promote Earth's surface deformation and stress conditions in and around volcanoes and fault zones.

Dr. Gonzalez acted twice as a Guest Editor of the Pure and Applied Geophysics journal. He is currently an Associate Editor of the Remote Sensing in Earth System Sciences and Royal Society Open Science journals. 